PROCEEDINGS OF THE

AMERICAN MATHEMATICAL SOCIETY

Volume 137, Number 3, March 2009, Pages 937-944

S 0002-9939(08)09725-6

Article electronically published on October 10, 2008

\title{
A SIMPLIFIED CALCULATION FOR THE FUNDAMENTAL SOLUTION TO THE HEAT EQUATION ON THE HEISENBERG GROUP
}

\author{
ALBERT BOGGESS AND ANDREW RAICH
}

(Communicated by Mei-Chi Shaw)

\begin{abstract}
Let $\mathcal{L}_{\gamma}=-\frac{1}{4}\left(\sum_{j=1}^{n}\left(X_{j}^{2}+Y_{j}^{2}\right)+i \gamma T\right)$ where $\gamma \in \mathbb{C}$, and $X_{j}$, $Y_{j}$ and $T$ are the left-invariant vector fields of the Heisenberg group structure for $\mathbb{R}^{n} \times \mathbb{R}^{n} \times \mathbb{R}$. We explicitly compute the Fourier transform (in the spatial variables) of the fundamental solution of the heat equation $\partial_{s} \rho=-\mathcal{L}_{\gamma} \rho$. As a consequence, we have a simplified computation of the Fourier transform of the fundamental solution of the $\square_{b}$-heat equation on the Heisenberg group and an explicit kernel of the heat equation associated to the weighted $\bar{\partial}$-operator in $\mathbb{C}^{n}$ with weight $\exp \left(-\tau P\left(z_{1}, \ldots, z_{n}\right)\right)$, where $P\left(z_{1}, \ldots, z_{n}\right)=\frac{1}{2}\left(\left|\operatorname{Im} z_{1}\right|^{2}+\right.$ $\left.\cdots+\left|\operatorname{Im} z_{n}\right|^{2}\right)$ and $\tau \in \mathbb{R}$.
\end{abstract}

\section{INTRODUCTION}

The purpose of this note is to present a simplified calculation of the Fourier transform of the fundmental solution of the $\square_{b}$-heat equation on the Heisenberg group. The Fourier transform of the fundamental solution has been computed by a number of authors Gav77, Hul76, CT00, Tie06. We use the approach of CT00, Tie06 and compute the heat kernel using Hermite functions but differ from the earlier approaches by working on a different, though biholomorphically equivalent, version of the Heisenberg group. The simplification in the computation occurs because the differential operators on this equivalent Heisenberg group take on a simpler form. Moreover, in the proof of Theorem 1.2, we reduce the $n$-dimensional heat equation to a 1-dimensional heat equation, and this technique would also be useful when analyzing the heat equation on the nonisotropic Heisenberg group (e.g., see [CT00]). We actually use the same version of the Heisenberg group as Hulanicki Hul76, but he computes the fundamental solution of the heat equation associated to the sub-Laplacian and not the Kohn Laplacian acting on $(0, q)$-forms.

A consequence of our fundamental solution computation is that we can explicitly compute the heat kernel associated to the weighted $\bar{\partial}$-problem in $\mathbb{C}^{n}$ when the weight is given by $\exp \left(-\tau P\left(z_{1}, \ldots, z_{n}\right)\right)$, where $\tau \in \mathbb{R}$ and $P\left(z_{1}, \ldots, z_{n}\right)=$ $\frac{1}{2}\left(\left|\operatorname{Im} z_{1}\right|^{2}+\cdots+\left|\operatorname{Im} z_{n}\right|^{2}\right)$. When $n=1$ and $p\left(z_{1}\right)$ is a subharmonic, nonharmonic

Received by the editors November 27, 2007.

2000 Mathematics Subject Classification. Primary 32W30, 33C45, 42C10.

Key words and phrases. Heisenberg group, heat equation, fundamental solution, heat kernel, Kohn Laplacian.

(C)2008 American Mathematical Society Reverts to public domain 28 years from publication 
polynomial, the weighted $\bar{\partial}$-problem (with weight $\exp \left(-p\left(z_{1}\right)\right)$ ) and the explicit construction of Bergman and Szegö kernels have been studied by a number of authors in different contexts (for example, see [Chr91, Has94, Has95, Has98, FS91, Ber92]). In addition, Raich has estimated the heat kernel and its derivatives Rai06b, Rai06a, Rai07, Rai.

\section{The Heisenberg Group And The $\square_{b}$-HeAt EQuation}

Definition 1.1. The Heisenberg group is the set $\mathbb{H}^{n}=\mathbb{R}^{n} \times \mathbb{R}^{n} \times \mathbb{R}$ with the following group structure:

$$
g * g^{\prime}=(x, y, t) *\left(x^{\prime}, y^{\prime}, t^{\prime}\right)=\left(x+x^{\prime}, y+y^{\prime}, t+t^{\prime}+x \cdot y^{\prime}\right),
$$

where $(x, y, t),\left(x^{\prime}, y^{\prime}, t^{\prime}\right) \in \mathbb{R}^{n} \times \mathbb{R}^{n} \times \mathbb{R}$ and $\cdot$ denotes the standard dot product in $\mathbb{R}^{n}$.

The left-invariant vector fields for this group structure are:

$$
X_{j}^{g}=\frac{\partial}{\partial x_{j}}+y_{j} \frac{\partial}{\partial t} \text { and } Y_{j}^{g}=\frac{\partial}{\partial y_{j}}, \quad 1 \leq j \leq n, \text { and } T^{g}=\frac{\partial}{\partial t} .
$$

The Heisenberg group can also be identified with the following hypersurface in $\mathbb{C}^{n+1}: H^{n}=\left\{\left(z_{1}, \ldots, z_{n+1}\right) \in \mathbb{C}^{n+1}: \operatorname{Im} z_{n+1}=(1 / 2) \sum_{j=1}^{n}\left(\operatorname{Im} z_{j}\right)^{2}\right\}$, where we identify $\left(z_{1}, \ldots, z_{n}, t+i(1 / 2) \sum_{j=1}^{n}\left(\operatorname{Im} z_{j}\right)^{2}\right) \in H^{n}$ with $\left(z_{1}, \ldots, z_{n}, t\right)=\left(x_{1}, \ldots\right.$, $\left.x_{n}, y_{1}, \ldots, y_{n}, t\right)$ where $z_{j}=x_{j}+i y_{j} \in \mathbb{C}$. With this identification, the left-invariant vector fields of types $(0,1)$ and $(1,0)$, respectively, are:

$$
\bar{Z}_{j}^{g}=\frac{1}{2}\left(X_{j}+i Y_{j}\right)=\frac{\partial}{\partial \bar{z}_{j}}+\frac{y_{j}}{2} \frac{\partial}{\partial t}, \quad Z_{j}^{g}=\frac{1}{2}\left(X_{j}-i Y_{j}\right)=\frac{\partial}{\partial z_{j}}+\frac{y_{j}}{2} \frac{\partial}{\partial t}
$$

for $g=(x, y, t) \in \mathbb{H}^{n}$ and $1 \leq j \leq n$.

The heat equation. The Kohn Laplacian $\square_{b}$ acting on $(0, q)$-forms on $H^{n} \approx$ $\mathbb{H}^{n}$ can be easily described in terms of these left-invariant vector fields. Suppose $f=\sum_{J \in \mathcal{I}_{q}} f_{J} d \bar{z}_{J}$ is a $(0, q)$-form where $\mathcal{I}_{q}$ is the set of all increasing $q$-tuples $J=\left(j_{1}, \ldots, j_{q}\right), 1 \leq j_{k} \leq n$. Then

$$
\square_{b} f=\sum_{J \in \mathcal{I}_{q}} \mathcal{L}_{n-2 q} f_{J} d \bar{z}_{J}
$$

where

$$
\mathcal{L}_{\gamma}=-\frac{1}{4}\left(\sum_{j=1}^{n}\left(X_{j}^{2}+Y_{j}^{2}\right)+i \gamma T\right) .
$$

See Stein ( Ste93, XIII §2) for details on computing $\square_{b}$. For comparison, the box operator (or Laplacian) in Hulanicki $(\underline{\text { Hul76] }})$ is $-\frac{1}{2} \sum_{j=1}^{n}\left(X_{j}^{2}+Y_{j}^{2}\right)$.

The heat equation is defined on $(0, q)$-forms $\rho$ on $\mathbb{H}^{n}$ with coefficient functions that depend on $s \in(0, \infty)$ and $(x, y, t) \in \mathbb{H}^{n}$. It is

$$
\frac{\partial \rho}{\partial s}=-\square_{b} \rho
$$

(note that here $s$ is the "time" variable and $t$ is a spatial variable). Since $\square_{b}$ acts diagonally, we can restrict ourselves to a fixed component and look for a fundamental 
solution $\rho$ that satisfies

$$
\left\{\begin{array}{l}
\frac{\partial \rho}{\partial s}=-\mathcal{L}_{\gamma} \rho \text { for } s>0,(x, y, t) \in \mathbb{H}^{n}, \\
\rho(s=0, x, y, t)=\delta_{0}(x, y, t)
\end{array}\right.
$$

(i.e., the delta function at the origin in the spatial variables).

Fourier transformed variables. We will use a Fourier transform in the spatial $(x, y, t)$ variables (i.e., not the $s$-variable): let $(\alpha, \beta, \tau)$ be the transform variables corresponding to $(x, y, t)$, and define:

$$
\widehat{f}(\alpha, \beta, \tau)=\int_{\mathbb{H}^{n}} f(x, y, t) e^{-i(\alpha \cdot x+\beta \cdot y+\tau t)} d x d y d t .
$$

Our main result is the following:

Theorem 1.2. For any $\gamma \in \mathbb{C}$, the spatial Fourier transform of the fundamental solution to the heat equation (2) is given by

$$
\hat{\rho}^{\gamma}(s, \alpha, \beta, \tau)=\frac{e^{-\gamma s \tau / 4}}{(\cosh (s \tau / 2))^{n / 2}} e^{-A\left(|\alpha|^{2}+|\beta|^{2}\right) / 2+i B \alpha \cdot \beta},
$$

where

$$
A=\frac{\sinh (s \tau / 2)}{\tau \cosh (s \tau / 2)}, \quad B=\frac{2 \sinh ^{2}(s \tau / 4)}{\tau \cosh (s \tau / 2)} .
$$

Note that $\gamma$ may be any complex number, but $\gamma=n-2 q$ is the value where $\mathcal{L}_{\gamma}$ corresponds to $\square_{b}$ on $(0, q)$-forms.

We also seek the fundamental solution to the heat equation associated to the weighted $\bar{\partial}$ operator in $(s, x, y)$-space. Given a function $f$ on $\mathbb{R}^{n} \times \mathbb{R}^{n} \times \mathbb{R}$, let

$$
\tilde{f}_{\tau}(x, y)=\int_{\mathbb{R}} e^{-i \tau t} f(x, y, t) d t
$$

be the partial Fourier transform in $t$. Define

$\bar{L}_{j}=\frac{\partial}{\partial \bar{z}_{j}}+\frac{i}{2} y_{j} \tau=\frac{1}{2}\left(\frac{\partial}{\partial x_{j}}+i \frac{\partial}{\partial y_{j}}+i y \tau\right), \quad L_{j}=\frac{\partial}{\partial z_{j}}+\frac{i}{2} y_{j} \tau=\frac{1}{2}\left(\frac{\partial}{\partial x_{j}}-i \frac{\partial}{\partial y_{j}}+i y \tau\right)$.

Note that these operators are just the Fourier transform of $\bar{Z}_{j}$ and $Z_{j}$ in the $t$ direction. If $\triangle_{x, y}$ is the Laplacian in both the $x$ and $y$ variables, the partial $t$-Fourier transform of $\mathcal{L}_{\gamma}$ is

$$
\tilde{\mathcal{L}_{\gamma}}=-\frac{1}{4}\left(\triangle_{x, y}+2 i \tau y \cdot \nabla_{x}-\left(\tau^{2} y \cdot y+\gamma \tau\right)\right) .
$$

The operator $\tilde{\mathcal{L}_{\gamma}}$ acts on functions, but it can be extended to $(0, q)$-forms by acting on each component function of the form. If $\gamma=n-2 q$, then $\tilde{\mathcal{L}_{\gamma}}$ is the higher dimensional analog of the $\square_{\tau p}$-operator from Rai06a, Rai07, Rai] associated to the weighted $\bar{\partial}$ operator in $\mathbb{C}^{n}$ with weight $\exp \left(-\tau P\left(z_{1}, \ldots, z_{n}\right)\right)$, where $P\left(z_{1}, \ldots, z_{n}\right)=$ $\frac{1}{2}\left(\left|\operatorname{Im} z_{1}\right|^{2}+\cdots+\left|\operatorname{Im} z_{n}\right|^{2}\right)$ and $\tau \in \mathbb{R}$. As a corollary to our main theorem, we compute the fundamental solution to the heat operator associated to this weighted $\bar{\partial}$. 
Corollary 1.3. For any $\gamma \in \mathbb{C}, \tau \in \mathbb{R}$, the function

$$
\tilde{\rho}_{\tau}^{\gamma}(s, x, y)=\frac{e^{-\gamma s \tau / 4}}{(2 \pi)^{n}(\cosh (s \tau / 2))^{n / 2}\left(A^{2}+B^{2}\right)^{n / 2}} e^{-\frac{A}{2\left(A^{2}+B^{2}\right)}\left(|x|^{2}+|y|^{2}\right)-i \frac{B}{A^{2}+B^{2}} x \cdot y}
$$

is the fundamental solution to the weighted $\bar{\partial}$ heat equation: $\left(\frac{\partial}{\partial s}+\tilde{\mathcal{L}}_{\gamma}\right) \tilde{\rho}_{\tau}^{\gamma}(s, x, y)=0$ with $\tilde{\rho}_{\tau}^{\gamma}(s=0, x, y)=\delta_{(0,0)}(x, y)$.

Finally, we use $\tilde{\rho}_{\tau}^{\gamma}$ to derive the heat kernel, as studied in Rai06a, Rai07, Rai, NS01.

Corollary 1.4. For any $\gamma \in \mathbb{C}, \tau \in \mathbb{R}$, let

$H_{\tau}^{\gamma}\left(s, x, y, x^{\prime}, y^{\prime}\right)=\frac{\tau^{n} e^{-\gamma s \tau / 4}}{(4 \pi)^{n} \sinh ^{n}(s \tau / 4)} e^{-\frac{\tau}{4} \operatorname{coth}(s \tau / 4)\left(\left|x-x^{\prime}\right|^{2}+\left|y-y^{\prime}\right|^{2}\right)-i \frac{\tau}{2}\left(x-x^{\prime}\right) \cdot\left(y+y^{\prime}\right)}$.

Then $H_{\tau}^{\gamma}$ is the heat kernel which satisfies the following property: if $f \in L^{2}(\mathbb{C})$, then

$$
H_{\tau}^{\gamma}[f](s, x, y)=\int_{\mathbb{R}^{n} \times \mathbb{R}^{n}} H_{\tau}^{\gamma}\left(s, x, y, x^{\prime}, y^{\prime}\right) f\left(x^{\prime}, y^{\prime}\right) d x^{\prime} d y^{\prime}
$$

is a solution to the following initial value problem for the heat equation:

$$
\left\{\begin{array}{l}
\left(\frac{\partial}{\partial s}+\tilde{\mathcal{L}_{\gamma}}\right) H_{\tau}^{\gamma}[f]=0 \\
H_{\tau}^{\gamma}[f](s=0, x, y)=f(x, y) .
\end{array}\right.
$$

Note that $H_{\tau}^{\gamma}$ is conjugate symmetric in $z=x+i y$ and $z^{\prime}=x^{\prime}+i y^{\prime}$ (i.e., switching $z$ with $z^{\prime}$ results in a conjugate).

\section{Proof of Theorem 1.2}

It is easy to verify the following calculations. Recall that ${ }^{\wedge}$ refers to the spatial Fourier transform

$$
\begin{aligned}
\widehat{X_{j}^{2}} f(\alpha, \beta, \tau) & =\left(-\alpha_{j}^{2}-2 i \alpha_{j} \tau \frac{\partial}{\partial \beta_{j}}+\tau^{2} \frac{\partial^{2}}{\partial \beta_{j}^{2}}\right) \widehat{f} \\
\widehat{Y_{j}^{2} f}(\alpha, \beta, \tau) & =-\beta_{j}^{2} \widehat{f} \\
\widehat{T f}(\alpha, \beta, \tau) & =i \tau \widehat{f} .
\end{aligned}
$$

We first reduce the problem down to dimension one. Define $\hat{\rho}^{\gamma, 1}$ by the same formula as given in (3), but for dimension one (i.e., $n=1$ and $\alpha, \beta \in \mathbb{R}$ ). From (3), note that

$$
\hat{\rho}^{\gamma}(s, \alpha, \beta, \tau)=\prod_{j=1}^{n} \hat{\rho}^{\gamma / n, 1}\left(s, \alpha_{j}, \beta_{j}, \tau\right), \quad \alpha=\left(\alpha_{1}, \ldots, \alpha_{n}\right), \beta=\left(\beta_{1}, \ldots, \beta_{n}\right) \in \mathbb{R}^{n}
$$

(note the $\gamma$ on the left and the $\gamma / n$ on the right). Once we show that $\rho^{\gamma, 1}$ satisfies the transformed heat equation in dimension one, i.e.,

$$
\left(\frac{\partial}{\partial s}-(1 / 4)\left(\widehat{X^{2}}+\widehat{Y^{2}}+i \gamma \widehat{T}\right)\right)\left\{\widehat{\rho}^{\gamma, 1}(s, \cdot, \cdot)\right\}=0
$$

with initial condition $\hat{\rho}^{\gamma, 1}(s=0, \cdot, \cdot, \cdot)=1$ (the Fourier transform of the delta function), then by using (5), it is an easy exercise to show that $\widehat{\rho}^{\gamma}$ in dimension $n$ satisfies Theorem 1.2 . 
From now on, we assume the dimension $n$ is one and so $x, y, \alpha$ and $\beta$ are all real variables. Also, $\gamma$ will be suppressed as a superscript. Define

$$
u(s, \alpha, \beta, \tau)=\widehat{\rho}(s, \alpha, \beta, \tau) e^{-i \frac{\alpha \beta}{\tau}} .
$$

Then, the following equations are easily verified:

$$
\begin{aligned}
u(s=0, \alpha, \beta, \tau) & =e^{-i \frac{\alpha \beta}{\tau}} \\
\frac{\partial u}{\partial s} & =\frac{1}{4}\left(\tau^{2} \frac{\partial^{2}}{\partial \beta^{2}}-\beta^{2}-\gamma \tau\right) u .
\end{aligned}
$$

The first equation follows from the fact that the Fourier transform of the delta function is the constant one. The second equation follows from the heat equation for $\widehat{\rho}$ (from (6) ) and the above formulas for the transformed differential operators $\widehat{X}, \widehat{Y}$ and $\widehat{T}$. We will refer to the above differential equation as the transformed heat equation.

Solution of heat equation using Hermite special functions. For $m=0,1,2$, $\ldots$ and $x \in \mathbb{R}$, let

$$
\psi_{m}(x)=\frac{(-1)^{m}}{\sqrt{2^{m} m ! \sqrt{\pi}}} e^{x^{2} / 2} \frac{d^{m}}{d x^{m}}\left\{e^{-x^{2}}\right\}
$$

For $\tau \in \mathbb{R}$, let

$$
\Psi_{m}^{\tau}(x)=|\tau|^{-1 / 4} \psi_{m}(x / \sqrt{|\tau|}) .
$$

It is a fact that $\psi_{m}$ and hence $\Psi_{m}^{\tau}$ form an orthonormal system for $L^{2}(\mathbb{R})$ (see [Tha93, pp. 1-7). It is also a fact (again see [Tha93, (1.1.28)) that

$$
\psi_{m}^{\prime \prime}(x)=x^{2} \psi_{m}(x)-(2 m+1) \psi_{m}(x) .
$$

We first assume that $\tau>0$ and later indicate the minor changes needed in the case that $\tau \leq 0$. Replacing $x$ by $\beta / \sqrt{\tau}$ in the previous equation yields:

$$
\left(\tau^{2} \frac{\partial^{2}}{\partial \beta^{2}}-\beta^{2}-\gamma \tau\right)\left\{\Psi_{m}^{\tau}\right\}(\beta)=-(2 m+1+\gamma) \tau \Psi_{m}^{\tau}(\beta) .
$$

In other words, $\Psi_{m}^{\tau}$ is an eigenfunction of the differential operator on the right side of (9) with eigenvalue $-\frac{1}{4}(2 m+1+\gamma) \tau$.

Since $\left\{\Psi_{m}^{\tau}\right\}$ are an orthonormal basis for $L^{2}(\mathbb{R}), u$ can be expressed as

$$
u(s, \alpha, \beta, \tau)=\sum_{m=0}^{\infty} a_{m}(\alpha, \tau) e^{-\frac{1}{4}(2 m+1+\gamma) s \tau} \Psi_{m}^{\tau}(\beta),
$$

where $a_{m}(\alpha, \tau)$ will be determined later. Differentiating this with respect to $s$ and using (10) gives

$$
\begin{aligned}
\frac{\partial}{\partial s} u(s, \alpha, \beta, \tau) & =\sum_{m=0}^{\infty} a_{m}(\alpha, \tau) e^{-\frac{1}{4}(2 m+1+\gamma) s \tau}\left(-\frac{1}{4}(2 m+1+\gamma)\right) \tau \Psi_{m}^{\tau}(\beta) \\
& =\frac{1}{4}\left(\tau^{2} \frac{\partial^{2}}{\partial \beta^{2}}-\beta^{2}-\gamma \tau\right)\{u(t, \alpha, \beta, \tau)\} .
\end{aligned}
$$


So, $u$ satisfies the transformed heat equation (9). To satisfy the initial condition (8), we must have

$$
e^{-i \alpha \beta / \tau}=u(s=0, \alpha, \beta, \tau)=\sum_{m=0}^{\infty} a_{m}(\alpha, \tau) \Psi_{m}^{\tau}(\beta) .
$$

Using the fact that the $\Psi_{m}^{\tau}(\beta)$ form an orthonormal system, we have

$$
a_{m}(\alpha, \tau)=\int_{\mathbb{R}} e^{-i \alpha \beta / \tau} \Psi_{m}^{\tau}(\beta) d \beta=\tau^{1 / 4} \int_{\mathbb{R}} e^{-i \frac{\alpha}{\sqrt{\tau}} \beta} \psi_{m}(\beta) d \beta .
$$

The integral on the right is just the Fourier transform of $\psi_{m}$ at the point $\alpha / \sqrt{\tau}$. From Thangavelu (Tha93, Lemma 1.1.3), the Fourier transform of $\psi_{m}$ equals $\psi_{m}$ up to a constant factor of $(-i)^{m} \sqrt{2 \pi}$. Therefore,

$$
a_{m}(\alpha, \tau)=(-i)^{m}(2 \pi)^{\frac{1}{2}} \tau^{\frac{1}{4}} \psi_{m}(\alpha / \sqrt{\tau}) .
$$

Substituting this value of $a_{m}$ into the expression for $u$ and rearranging gives:

$$
u(s, \alpha, \beta, \tau)=(2 \pi)^{1 / 2} e^{-\frac{1}{4}(1+\gamma) s \tau} \sum_{m=0}^{\infty}(-i)^{m} \psi_{m}\left(\frac{\alpha}{\sqrt{\tau}}\right) \psi_{m}\left(\frac{\beta}{\sqrt{\tau}}\right) e^{-\frac{1}{2} m s \tau} .
$$

Now solving for $\hat{\rho}$ (see equation (7)) yields

$$
\begin{aligned}
\hat{\rho}(s, \alpha, \beta, \tau) & =e^{i \alpha \beta / \tau} u(s, \alpha, \beta, \tau) \\
& =(2 \pi)^{\frac{1}{2}} e^{-\frac{1}{4}(1+\gamma) s \tau} \sum_{m=0}^{\infty}(-i)^{m} \psi_{m}\left(\frac{\alpha}{\sqrt{\tau}}\right) \psi_{m}\left(\frac{\beta}{\sqrt{\tau}}\right) e^{-\frac{1}{2} m s \tau} e^{i \alpha \beta / \tau} .
\end{aligned}
$$

Now let $S=e^{-s \tau / 2}, x=\alpha / \sqrt{\tau}, y=\beta / \sqrt{\tau}$. Since $|i S|<1$, we obtain (see [Tha93], $(1.1 .36))$

$$
\begin{aligned}
\hat{\rho}(s, \alpha, \beta, \tau) & =(2 \pi)^{\frac{1}{2}} S^{\frac{1}{2}(1+\gamma)}\left(\sum_{m=0}^{\infty}(-i S)^{m} \psi_{m}(x) \psi_{m}(y)\right) e^{i x y} \\
& =\frac{\sqrt{2} S^{\frac{1}{2}(1+\gamma)}}{\left(1+S^{2}\right)^{\frac{1}{2}}} e^{-\frac{1}{2} \frac{1-S^{2}}{1+S^{2}}\left(x^{2}+y^{2}\right)} e^{i x y\left(\frac{-2 S}{1+S^{2}}+1\right)} .
\end{aligned}
$$

Now substituting in for $S, x$ and $y$, a short calculation finishes the proof for $\tau>0$. Note that $\hat{\rho}(s=0, \alpha, \beta, \tau)=1$ (the Fourier transform of the delta function at the origin).

When $\tau=0$, the solution in (3) becomes $\widehat{\rho}(s, \alpha, \beta)=e^{-s\left(\alpha^{2}+\beta^{2}\right) / 4}$, which is easily shown to satisfy (6).

If $\tau<0$, then $\tau$ is replaced by $|\tau|$ on the right side of (10), which slightly changes the subsequent calculations. However the formula for the solution given Theorem 1.2 remains valid for $\tau<0$.

\section{Proof of the corollaries}

Proof of Corollary 1.3. Again, we assume the dimension is $n=1$. The fundamental solution to this heat operator must satisfy

$$
\frac{\partial}{\partial s} \tilde{\rho}_{\tau}(s, x, y)+\tilde{\mathcal{L}}_{\gamma} \tilde{\rho}_{\tau}=0
$$

with the initial condition $\tilde{\rho}_{\tau}(s=0, x, y)=\delta_{0}(x, y)$. Now since $\hat{\rho}$ is the Fourier transform of the fundamental solution to the original heat operator, clearly $\tilde{\rho}_{\tau}$ can 
be obtained by taking the inverse Fourier transform of $\hat{\rho}$ in the $\alpha, \beta$ variables. This is a standard calculation involving Gaussian integrals and will be left to the reader.

Proof of Corollary 1.4. If $L_{j}$ and $\bar{L}_{j}, 1 \leq j \leq n$, had constant coefficients, then the heat kernel would just be $\tilde{\rho}_{\tau}\left(s, x-x^{\prime}, y-y^{\prime}\right)$, an ordinary convolution. However, we must multiply by a "twist" factor $e^{-i \tau\left(x-x^{\prime}\right) \cdot y^{\prime}}$ to account for the fact that $L_{j}$ and $\overline{L_{j}}$ have variable coefficients. Let

$$
H_{\tau}\left(s, x, y, x^{\prime}, y^{\prime}, \tau\right)=\tilde{\rho}_{\tau}\left(s, x-x^{\prime}, y-y^{\prime}\right) e^{-i \tau\left(x-x^{\prime}\right) \cdot y^{\prime}} .
$$

Note that $H_{\tau}(f)$ satisfies the initial condition given in (4) in view of the initial condition satisfied by $\tilde{\rho}_{\tau}$ and noting that the twist term is 1 at $x^{\prime}=x$. Showing that $H_{\tau}$ satisfies the heat equation in the $s, x, y$ variables is a short calculation that uses the equation

$$
\begin{aligned}
\left(\frac{\partial}{\partial s}-\frac{1}{4}\left(\triangle_{x, y}+2 i \tau\left(y-y^{\prime}\right) \cdot \nabla_{x}-\left(\tau^{2}\left(y-y^{\prime}\right)\right.\right.\right. & \left.\left.\left.\cdot\left(y-y^{\prime}\right)+\gamma \tau\right)\right)\right) \\
& \times\left\{\tilde{\rho}_{\tau}\left(s, x-x^{\prime}, y-y^{\prime}\right)\right\}=0,
\end{aligned}
$$

which is just the equation $\left(\frac{\partial}{\partial s}+\tilde{\mathcal{L}}_{\gamma}\right) \tilde{\rho}_{\tau}=0$ at the point $\left(s, x-x^{\prime}, y-y^{\prime}\right)$.

Simplification of the formula for $H_{\tau}$. Note that the coefficient of the imaginary part of the exponent of $\tilde{\rho}_{\tau}$ is

$$
\frac{-B}{A^{2}+B^{2}} \quad \text { where } \quad A=\frac{\sinh (s \tau / 2)}{\tau \cosh (s \tau / 2)}, \quad B=\frac{2 \sinh ^{2}(s \tau / 4)}{\tau \cosh (s \tau / 2)} .
$$

An easy calculation with cosh and sinh identities shows that

$$
\frac{B}{A^{2}+B^{2}}=\frac{\tau}{2} \quad \text { and } \quad \frac{A}{B}=\frac{\cosh (s \tau / 4)}{\sinh (s \tau / 4)} .
$$

Consequently, the fundamental solution $H_{\tau}$, from (11) and Corollary 1.3, can be rewritten

$H_{\tau}\left(s, x, y, x^{\prime}, y^{\prime}\right)=\frac{\tau^{n} e^{-\gamma s \tau / 4}}{(4 \pi)^{n} \sinh ^{n}(s \tau / 4)} e^{-\frac{\tau}{4} \operatorname{coth}(s \tau / 4)\left(\left|x-x^{\prime}\right|^{2}+\left|y-y^{\prime}\right|^{2}\right)-i \frac{\tau}{2}\left(x-x^{\prime}\right) \cdot\left(y+y^{\prime}\right)}$.

\section{REFERENCES}

[Ber92] B. Berndtsson. Weighted estimates for $\bar{\partial}$ in domains in $\mathbb{C}$. Duke Math. J., 66(2):239-255, 1992. MR:1162190 (93f:32018)

[CT00] Der-Chen Chang and Jingzhi Tie. Estimates for powers of the sub-Laplacian on the non-isotropic Heisenberg group. J. Geom. Anal., 10:653-678, 2000. MR.1817779 (2001m:58047)

[Chr91] M. Christ. On the $\bar{\partial}$ equation in weighted $L^{2}$ norms in $\mathbb{C}^{1} . J$. Geom. Anal., 1(3):193-230, 1991. MR.1120680 (92j:32066)

[FS91] J.E. Fornæss and N. Sibony. On $L^{p}$ estimates for $\bar{\partial}$. In Several Complex Variables and Complex Geometry, Part 3 (Santa Cruz, CA, 1989), Proc. Sympos. Pure Math., 52, Part 3, pages 129-163, American Mathematical Society, Providence, R.I., 1991. MR.1128589 (92h:32030)

[Gav77] B. Gaveau. Principe de moindre action, propogation de la chaleur et estimées sous elliptiques sur certains groupes nilpotents. Acta Math., 139:95-153, 1977. MR0461589 $(57: 1574)$ 
[Has94] F. Haslinger. Szegö kernels for certain unbounded domains in $\mathbb{C}^{2}$. Travaux de la Conférence Internationale d'Analyse Complexe et du 7e Séminaire Roumano-Finlandais (1993). Rev. Roumaine Math. Pures Appl., 39:939-950, 1994. MR1406110 (97f:32026)

[Has95] F. Haslinger. Singularities of the Szegö kernel for certain weakly pseudoconvex domains in $C^{2}$. J. Funct. Anal., 129:406-427, 1995. MR.1327185 (96g:32045)

[Has98] F. Haslinger. Bergman and Hardy spaces on model domains. Illinois J. Math., 42:458469, 1998. MR 1631252 (99f:32004)

[Hul76] A. Hulanicki. The distribution of energy in the Brownian motion in the Gaussian field and analytic-hypoellipticity of certain subelliptic operators on the Heisenberg group. Studia Math., 56:165-173, 1976. MR0418257 (54:6298)

[NS01] A. Nagel and E.M. Stein. The $\square_{b}$-heat equation on pseudoconvex manifolds of finite type in $\mathbb{C}^{2}$. Math. Z., 238:37-88, 2001. MR.1860735(2002h:32031)

[Rai] Andrew Raich. Heat equations and the weighted $\bar{\partial}$-problem with decoupled weights, submitted. arXiv:0704.2768.

[Rai06a] Andrew Raich. Heat equations in $\mathbb{R} \times \mathbb{C}$. J. Funct. Anal., 240(1):1-35, 2006. MR2259891 (2007h:32059)

[Rai06b] Andrew Raich. One-parameter families of operators in C. J. Geom. Anal., 16(2):353-374, 2006. MR2223806 (2007c:32049)

[Rai07] Andrew Raich. Pointwise estimates for relative fundamental solutions for heat equations in $\mathbb{R} \times \mathbb{C}$. Math. Z., 256:193-220, 2007. MR2282265 (2008b:32030)

[Ste93] Elias M. Stein. Harmonic Analysis: Real-Variable Methods, Orthogonality, and Oscillatory Integrals. Princeton Mathematical Series, 43. Princeton University Press, Princeton, New Jersey, 1993. MR1232192 (95c:42002)

[Tha93] Sundaram Thangavelu. Lectures on Hermite and Laguerre Expansions, Mathematical Notes, 42. Princeton University Press, Princeton, New Jersey, 1993. MR 1215939 (94i:42001)

[Tie06] Jingzhi Tie. The twisted Laplacian on $\mathbb{C}^{n}$ and the sub-Laplacian on $H_{n}$. Comm. Partial Differential Equations, 31:1047-1069, 2006. MR2254603 (2007e:32049)

Department of Mathematics, Texas A\&M University, Mailstop 3368, College Station, TeXas 77845-3368

E-mail address: boggess@math.tamu.edu

Department of Mathematical Sciences, 1 University of Arkansas, SCEN 327, Fayetteville, Arkansas 72701

E-mail address: araich@uark.edu 